\title{
Um Estudo Preliminar das Relações entre Características de Blockchain e a Aplicação na Sociedade
}

\author{
Emanuel F. Coutinho ${ }^{1}$, Wagner L. Braga Bezerra ${ }^{1}$, Delano Maia ${ }^{1}$ \\ ${ }^{1}$ Programa de Pós-Graduação em Computação (PCOMP) \\ Universidade Federal do Ceará (UFC) - Campus de Quixadá \\ emanuel.coutinho@ufc.br, wagnerbragacrfealu.ufc.br, delanomaia@ufc.br
}

\begin{abstract}
Blockchain is currently being widely discussed in academia and industry. Several areas are experiencing improvements in their processes and greater security in data management, such as health, education and finance. In this context, the objective of this work is to report initial studies of the relationships and impacts of blockchain on society, mainly due to applications in the most diverse areas. For this, an online questionnaire on blockchain aspects in society was applied, followed by a qualitative analysis to better understand the results. As preliminary results, health and finance are the areas that most use blockchain, and the questionnaire revealed that it is important to study the impacts of blockchain on society.
\end{abstract}

Resumo. Blockchain atualmente está sendo muito discutida na academia e indústria. Diversas áreas estão experimentando melhorias em seus processos e maior segurança na gestão dos dados, como saúde, educação e finanças. Nesse contexto, o objetivo deste trabalho é relatar estudos iniciais das relações e impactos da blockchain na sociedade, principalmente devido às aplicações nas mais diversas áreas. Para isso, um questionário online sobre aspectos de blockchain na sociedade foi aplicado, seguido de uma análise qualitativa para melhor compreender os resultados. Como resultados preliminares, saúde e finanças são as áreas que mais utilizam blockchain, e o questionário revelou que é importante estudar os impactos da blockchain na sociedade.

\section{Introdução}

Blockchain atualmente está sendo muito discutida tanto na academia quanto na indústria. E essa discussão está permeando todos os setores da sociedade. Um exemplo desse efeito é a proposição de projetos baseados em blockchain em algumas cidades, como Dubai, Estocolmo, Toronto e Visakhapatnam [Xie et al. 2019].

Blockchain possui alguns recursos que a tornam uma tecnologia atraente para enfrentar diversos desafios [Xie et al. 2019]: descentralização, pseudonimato, transparência, democracia, segurança e imutabilidade. Essas características possibilitam que diversas áreas melhorem seus processos e tenham uma maior segurança na gestão dos dados. Áreas como finanças, saúde, educação e logística estão se apropriando da blockchain para o incremento de suas atividades e aplicações. Assim, blockchain abriu uma série de possibilidades para empresas nas quais o valor pode ser transferido diretamente entre os participantes pela Internet, da mesma maneira fácil como pagar em dinheiro e conveniente que usar mensagens instantâneas sem intermediários centralizados [Aste et al. 2017]. 
No contexto da atual quarta revolução industrial, caracterizada pela fusão de diversas tecnologias que misturam o espaço físico e cibernético, a blockchain possui um papel mais amplo. Junto com outras tecnologias emergentes, como computação em névoa, aprendizado de máquina, inteligência artificial e veículos autônomos, a blockchain pode impactar muitos setores de negócios e a sociedade em geral [Aste et al. 2017].

Em geral, nossa sociedade é centralizada, com hierarquias institucionais para governar atividades de nossas comunidades socioeconômicas [Aste et al. 2017]. Nesse contexto, a blockchain possibilita novos modelos de negócios, novos processos de trabalho e de produção nos quais o acesso e o compartilhamento é sobre propriedade. E assim, a blockchain cria a oportunidade para a geração de um nível de confiança necessário entre partes desconhecidas e anônimas, permitindo a negociação sem intermediários.

Nesse contexto, esse trabalho tem como objetivo iniciar os estudos sobre as relações e impactos de blockchain sobre a sociedade, seja por se beneficiar de suas características, seja por aplicações nas mais diversas áreas. Para isso, aplicou-se um questionário online com algumas pessoas que já conhecem blockchain sobre aspectos da sociedade, seguido de uma análise qualitativa para melhor compreender os resultado. Os resultados servirão para justificar a continuidade da pesquisa e seu aprofundamento.

\section{Metodologia}

A metodologia deste trabalho consiste na execução de quatro etapas, dispostas a seguir: (i) Definição de perguntas de partida - identificação de uma visão geral inicial da sociedade, blockchain e aplicações; (ii) Questionário online - coleta de dados demográficos e das questões de partida; (iii) Consolidação dos dados - organização e interpretação inicial dos dados coletados; (iv) Análise qualitativa - utilização de recursos da Grounded Theory [Strauss and Corbin 2007] para uma análise qualitativa dos dados coletados; e (vi) Obtenção dos direcionamentos futuros - definição de tendências e perspectivas de pesquisa. O questionário online projetado está disposto na Tabela 1.

Tabela 1. Questões objetivas (QO) e questões abertas (QA) do questionário

\begin{tabular}{|l|l|}
\hline QO1 & Qual seu nível de conhecimento dos impactos da utilização de blockchain na sociedade? (Nada, Pouco, Razoável, Alto) \\
\hline QO2 & Você acha relevante pesquisas relacionadas a blockchain e sociedade? (Sim, Não) \\
\hline QA1 & Onde blockchain pode ser aplicada? \\
\hline QA2 & Como blockchain pode melhorar a vida das pessoas? \\
\hline QA3 & Qual o impacto da utilização de blockchain sobre aplicações tradicionais? \\
\hline
\end{tabular}

\section{Resultados}

A pesquisa teve 11 respondentes, todos pesquisadores em blockchain em algum nível. $\mathrm{O}$ objetivo desse questionário foi verificar a viabilidade da pesquisa e o interesse em saber das relações de blockchain e sociedade. Apesar de poucas respostas, as questões possibilitaram bons feedbacks, que colaborarão para o prosseguimento da pesquisa.

Em relação a Q01, 7 pessoas responderam que possuíam um nível de conhecimento dos impactos da utilização de blockchain na sociedade razoável, 3 responderam que o nível de conhecimento era pouco, e apenas 1 respondeu que o nível era alto. Para a Q02, todos responderam que sim, que pesquisas relacionadas com blockchain e sociedade são relevantes. Isso leva a crer que a pesquisa tem importância para ser aprofundada. 
A questão QA1 indicou que diversas áreas podem se beneficiar da blockchain. As áreas que foram citadas, por ordem decrescente de frequência, foram: Saúde (7), Finanças (4), Transportes (4), Educação (2), Logística (2), Cartórios (1), Governo (1), Indústria (1), Judiciário (1), Mercado (1) e Negócios (1). Um exemplo de comentário que refletiu bem essa questão foi o de P1: "Em todas as áreas! Finanças, educação, saúde, logística são apenas alguns exemplos". O destaque de finanças é para as criptomoedas, como bitcoin. Entretanto, saúde foi a área mais ressaltada, com registros eletrônicos de dados de saúde, como no discurso de P2 : "Em quase todos os âmbitos do meio social, quando se utiliza o conceito de tornar um processo seguro por meio de registro definitivo, pode-se usar blockchain. Temos exemplo de uso na saúde, negócios, veiculos, entre outros".

Na questão QA2, diversos benefícios da utilização de blockchain foram citados: Segurança (4), Imutabilidade (3), Eliminação de elementos terceiros (2), Rapidez (2), Redução de custos (2), Auditabilidade (1), Automação (1), Confiança (1), Controle de acesso (1), Descentralização (1), Disponibilidade (1), Integridade (1) e Transparência (1). Muitas dessas melhorias estão relacionadas às características básicas de blockchain. Muito foco para a segurança da informação, como o comentário de P3: "Provendo segurança, controle de acesso e disponibilidade de informações". Algumas respostas foram mais técnicas, mas reforçaram características fundamentais da blockchain, como a resposta de P2: "Blockchain pode fornecer garantias de integridade onde um informação, que muitas vezes pode ser crucial. Por exemplo transações bancárias usando blockchain vão garantir que o registro da transação está imutável, concreto, assim pagador e recebedor tem essas garantias". Um destaque foi a rapidez, associada à "desburocratização" de processos, devido à eliminação de terceiros e redução de custos. P10 e P11 destacaram esse ponto, respectivamente: "Importância ampla em indústrias, ajudando as empresas a trabalhar com mais inteligência e rapidez" e "Eliminar ou reduzir custos, como o exemplo dos serviços de cartório. Ajudar na transparência de dados e sistemas etc".

Por fim, QA3 destacou a melhoria que aplicações tradicionais teriam com a agregação das características de blockchain, sendo estes: Auditabilidade (3), Segurança (3), Imutabilidade (2), Adaptação (1), Confiança (1), Consenso (1), Descentralização (1), Desempenho (1), Dinamismo (1), Economia (1), Integrar (1), Interação (1), Irrefutabilidade (1), melhoria de processos (1), Aumento da qualidade (1), Surgimento de novos negócios (1) e Transparência (1). P1 relatou que aplicações tradicionais sempre existirão e a qualidade pode aumentar com blockchain: "Elas não vão deixar de existir nunca, mas com a agregação de recursos de blockchain quando possivel, a qualidade vai aumentar. Também tem que se considerar o desempenho de aplicações blockchain e quando ela pode ser utilizada". Os processos relacionados às aplicações também foram citados, como descrito por P2: "Melhoramento de processos. O blockchain traz uma maneira mais concreta e assertiva para realização dos mesmos processos. Um bom exemplo disso são os contratos inteligentes". E a adaptação, citada apenas por P3, é algo que terá que ocorrer na migração ou manutenção de aplicações tradicionais para blockchain: "Será necessário adaptação das aplicações tradicionais para incorporar ou integrar soluções baseadas em blockchain para ter os benefícios dessa nova tecnologia". Auditabilidade e segurança foram os mais citados, e são características de blockchain bastante conhecidas na academia e indústria, reforçado pela resposta de P11: "Uma gestão de dados mais confiável, com distribuição replicada totalmente e a possibilidade de auditabilidade. Surge como um tecnologia disruptiva que fornece uma série de características que podem ser essenciais: 
consenso, auditabilidade, irrefutabilidade etc".

\section{Discussão}

\subsection{Análise Qualitativa}

Para obter um melhor e mais profundo entendimento sobre os resultados das questões abertas, executou-se alguns procedimentos do Grounded Theory. A ideia era identificar categorias e relações. A Figura 1 apresenta o resultado da análise qualitativa. Os códigos identificados e suas respectivas frequências foram: conceito (20), áreas (11), benefício (8), opinião (7), ação (6) e impacto (5). Conceito significa características técnicas da blockchain ou elementos estruturais, e conhecimento teórico ou prático. Benefício é qualquer vantagem da blockchain para aplicações ou sociedade, sem detalhes técnico. Ação são atitudes que o respondente descreveu pelo uso da blockchain. Áreas são aplicações ou domínios onde blockchain pode ser utilizada. Impacto é uma consequência do uso da blockchain. Opinião representa algo mais pessoal que o respondente escreveu, como o sentimento pelo uso da blockchain. As relações identificadas foram apenas três: conceito está relacionado com áreas, conceito é causa de impacto, e conceito é causa de benefícios.

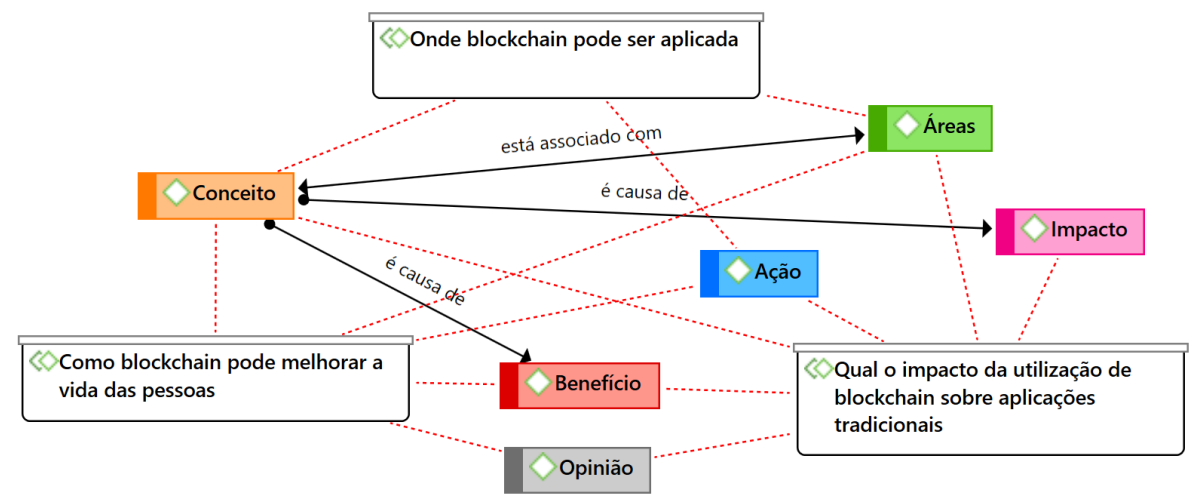

Figura 1. Relações e códigos da análise qualitativa com Grounded Theory

Para a relação conceito está relacionado com áreas identificou-se em vários comentários que toda vez que uma área era citada, geralmente havia alguma característica relacionada. Os comentários de $\mathbf{P 1 1}$ e $\mathbf{P 4}$ respectivamente corroboram essa relação: "Poderia substituir alguns serviços de cartório, consenso distribuído para disseminação de informação, auditabilidade de dados/sistemas, aplicações de laudos médicos, serviços de localização de objetos (transportadoras) etc" e "O blockchain acaba gerando mais transparência e segurança para os usuários...no caso de um contrato inteligente...oferece agilidade e um histórico completo de um imóvel, por exemplo, informações do tipo quantos donos já tiveram antes". A relação conceito é causa de impacto demonstrou que alguns respondentes possuíam conhecimento de conceitos de blockchain e conseguiam listar impactos. O comentário de $\mathbf{P 7}$ reforçou essa relação: "A blockchain pode impactar positivamente em diversas aplicações tradicionais que necessitam de suas características de imutabilidade, descentralização e segurança". Por fim, a relação conceito é causa de benefícios segue a mesma linha de raciocínio da relação anterior, pois de maneira geral, quem possui conhecimentos em blockchain também soube descrever benefícios de seu uso. $\mathbf{P 6}$ redigiu o seguinte comentário sobre essa relação:“Em eliminar os intermediários para baixar custos em diversos setores". 


\subsection{Direcionamentos Futuros da Pesquisa e Perspectivas}

Em relação às áreas de aplicação, saúde e finanças se destacaram mais. Motivado pelo bitcoin, a área relacionada a finanças, negociações e transações financeiras tende a se expandir bastante. Estudar aspectos humanos e tecnológicos dessa áreas pode ser um boa oportunidade de pesquisa. Na área da saúde, por exemplo, [Moreira Neto et al. 2020] apresentaram uma arquitetura de $e$-health com Internet das Coisas para a agregação de recursos de blockchain, com desenvolvimento de aplicações mobile e web. As perspectivas de expansão da pesquisa são boas e a integração das áreas tende a crescer.

As relações identificadas pela análise qualitativa possuem a categoria "conceito" envolvida. Assim, estudar conceitos de blockchain, suas características, como elas podem ser aplicadas nos sistemas atuais, seus impactos no desenvolvimento, como elas melhoram a qualidade das aplicações e dos usuários são aspectos a serem investigados.

Avaliar a viabilidade de se utilizar blockchain é um aspecto importante, pois há um custo computacional grande. Alguns trabalhos na literatura discutiram sobre esta relação, como [Pedersen et al. 2019], onde critérios para se decidir se usar blockchain vale a pena ou não. Esse tipo de validação é necessária para que as aplicações não sejam prejudicadas.

\section{Conclusão}

Esta pesquisa é um ensaio inicial sobre blockchain e seus impactos na sociedade. Observou-se que atualmente a tecnologia está ampliando sua utilização em diversas áreas, e que a sociedade está percebendo seus benefícios. Mesmo com poucas respostas no questionário, os resultados são um indício que a pesquisa deve prosseguir e ser aprofundada. Estes resultados são preliminares, apenas para sondar o interesse pela pesquisa, e que não teve participação de pessoas que não pesquisam em blockchain. A aplicação de um questionário mais elaborado com mais pessoas, grupos de pesquisa, academia e indústria é o próximo passo, atingindo o máximo de respondentes das mais diversas áreas. Como trabalhos futuros pretende-se ampliar o estudo, analisando o impacto social da tecnologia e sua relevância em áreas diversas, investindo nos direcionamentos futuros apresentados.

\section{Referências}

Aste, T., Tasca, P., and Di Matteo, T. (2017). Blockchain technologies: The foreseeable impact on society and industry. Computer, 50(9):18-28.

Moreira Neto, M., Coutinho, E. F., Moreira, L. O., and de Souza, J. N. (2020). Toward blockchain technology in iot applications: An analysis for e-health applications. In Internet of Things. A Confluence of Many Disciplines, pages 36-50, Cham. Springer International Publishing.

Pedersen, A. B., Risius, M., and Beck, R. (2019). A ten-step decision path to determine when to use blockchain technologies. MIS Quarterly Executive, 18(2).

Strauss, A. and Corbin, J. (2007). Basics of Qualitative Research: Techniques and Procedures for Developing Grounded Theory. SAGE Publications, 3rd edition.

Xie, J., Tang, H., Huang, T., Yu, F. R., Xie, R., Liu, J., and Liu, Y. (2019). A survey of blockchain technology applied to smart cities: Research issues and challenges. IEEE Communications Surveys \& Tutorials, 21(3):2794-2830. 